\title{
Peculiarities of Computer Simulation and Statistical Representation of Time-Spatial Metocean Fields
}

\author{
A. Boukhanovsky ${ }^{1}$, V. Rozhkov ${ }^{2}$, and A. Degtyarev ${ }^{1}$ \\ ${ }^{1}$ Institute for high performance computing and data bases \\ Fontanka 118, 198005 St.Petersburg, Russia \\ ${ }^{2}$ St.Petersburg branch of State Oceanographic Institute \\ 23 linia 2A, 199026 St.Petersburg, Russia \\ avb@fn.csa.ru, ocean@nwmet.nw.ru, deg@fn.csa.ru
}

\section{Introduction}

The integral property of hydrometeorological fields (atmospheric pressure, wind speed, wind waves, temperature and salinity of seawater, sea currents) is their spatialtime variability caused by superposition and interaction of a large number of factors. Characteristic ranges of variability are interannual variability (cyclicity of fluctuations more than one year), annual cycles, synoptic variability (time scale of fluctuations from one day to several days), daily cycles, small-scale fluctuations (cyclicity from several seconds to one hour). The presence of multiscale variability causes nonstationarity of hydrometeorological processes. Variety of active factors results in spatial heterogeneity of fields (stratification of surface layer and seawater, movement of baric formations in an atmosphere and mesoscale eddies in oceans and seas).

Traditionally the basis of objective laws of description of hydrometeorological field's variability is the analysis of full-scale data: shipboard observations during voyages, continuous observations at sea or coastal stations, satellite information. The intensive development of mathematical modeling methods on the basis of analytical and numerical solutions of system of termo- and hydrodynamics equations under the appropriate initial and boundary conditions has allowed to fill up considerably this information base. It results from both reproduction of the measurement data in points of a regular grid by means of reanalysis [12], and obtaining information about nonobservable parameters on verified models. As a result, for example, we have wind waves [13], termohaline structure of waters and ecological system parameters [9].

The specificity of application of hydrodynamic models for observation data assimilation in information base consists in obtaining results as a file of values $\Xi=\left\{\xi_{p}\right\}_{p=1}^{m}$ in different spatial points $\vec{r}\left(x_{i}, y_{j}, z_{k}\right)$ at the time moment $t_{s}$. Complexity, non-uniform scale, polycyclicity and great variability of the hydrometeorological information result in necessity to consider them as stochastic functions of time and spatial coordinates and to describe their properties in terms of probabilistic characteristics [16]. There are mean value $m_{\Xi}(\vec{r}, t)$, variance $D(\vec{r}, t)$, covariation function $K_{\Xi}(\vec{r}, \vec{\rho}, t, \tau)$ and spectral density $S_{\Xi}(\omega, \vec{r}, t)$, which depend on several variable (coordinates $r$ and time $t$, frequency $\omega$, spatial $\vec{\rho}$ and time $\tau$ shifts). Traditional problem of multivariate statistical analysis (MSA) is estimation of such characteristics on natural data. 
Classical MSA operates with concepts of multivariate stochastic value, system of dependent stochastic values and multivariate time series [2,4]. The purpose of the paper the use of MSA methods with regard for specificity of spatial-time fields obtained by hydrodynamic simulation.

\section{Hydrodynamic Simulation as Metocean Data Source}

The evolution of surface wave field in space and time is governed by the basic transport or energy balance equation [17]

$$
\frac{\partial S}{\partial t}+v \cdot \nabla S=G
$$

where $\mathrm{S}(\omega, \theta, \vec{r}, \mathrm{t})$ is the two-dimensional wave spectrum, dependent on frequency $\omega$ and propagation direction $\theta ; \mathrm{v}=\mathrm{v}(\omega, \theta)$ is the group velocity. $\mathrm{S}$ is net source function. It is represented as the sum of the input $S_{\mathrm{in}}$ by the wind, the nonlinear transfer $S_{\mathrm{nl}}$ by resonant wave-wave interaction, and the dissipation $\mathrm{S}_{\mathrm{d}}$. There are some other terms (interaction with slowly variable currents, etc.) which are normally small. They are not included in the propagation operator.

Equation (1) describes functional relation between fields of atmosphere pressure, wind and waves. There are many calculation models based on (1) devoted to obtaining time-spatial wave field. All they are differ from one another by sources function presentation and computational layout.

The first wave model which was realized as world famous software is WAM-model [17]. The theory and methods of numerical simulation are continuously improved. Now we have new results and models (WAVEWATCH [18], PHIDIAS [19], TOMAWAC [20], INTERPOL [21]) for deep and (SWAN [22]) for shallow water. Such results and a great activity in the field of reanalysis of pressure and wind in points of regular mesh $[12,13]$ give possibility to use results of numerical simulation of time-spatial fields as initial data for analysis by means of MSA.

Specific character of computer presentation of hydrometeorological fields information is large volume of used data and long time for calculation. Hence, application of high performance computers is necessary. Part of the results used in this paper for MSA was obtained in Institute for High Performance Computing on supercomputer HP SPP1600 (8 processors).

\section{Metocean Events as Elements of Functional Spaces}

The basic probability model for the analysis of the hydrometeorological data is the random function $\eta(\vec{r}, t)$ of spatial coordinates $\vec{r}$ and time $t$ characterized by mean value

$$
m_{\eta}(\vec{r}, t)=E[\eta(\vec{r}, t, w)]
$$

and covariance function

$$
K_{\eta}(\vec{r}, \vec{\rho}, t, \tau)=E\left[\eta^{o}(\vec{r}, t, w) \cdot \eta^{o}(\vec{r}+\vec{\rho}, t+\tau, w)\right]
$$


where $E[\bullet]$ is the operator of population mean (averaging on ensemble of realizations numbered by index $\mathrm{w}), \eta^{0}(\bullet)=\eta(\bullet)-m_{\eta}(\bullet)$.

By example of the three interconnected fields: atmospheric pressure $\mathrm{p}(\vec{r}, \mathrm{t})$, wind speed $\vec{V}(\vec{r}, \mathrm{t})$ and wind waves $\mathrm{S}(\omega, \Theta, \vec{r}, \mathrm{t})$. It is easy to make sure that the operations of addition and multiplication in (1) and (2) are subject to a concrete definition owing to specificity of fields $\mathrm{p}(\bullet), \vec{V}(\bullet), \mathrm{S}(\bullet)$. For each value $(\vec{r}, t)$ the field of pressure $\mathrm{p}(\bullet)$ is scalar value, the field is vectorial value depending on a gradient of scalar field $\mathrm{p}(\bullet)$, field of wind waves computed in accordance with field $\vec{V}(\bullet)$ through the equation of wave energy balance (1) is represented by frequency directional spectrum $S(\omega, \Theta, \bullet)$, where $\omega$ is frequency, $\Theta$ is mean direction of waves propagation.

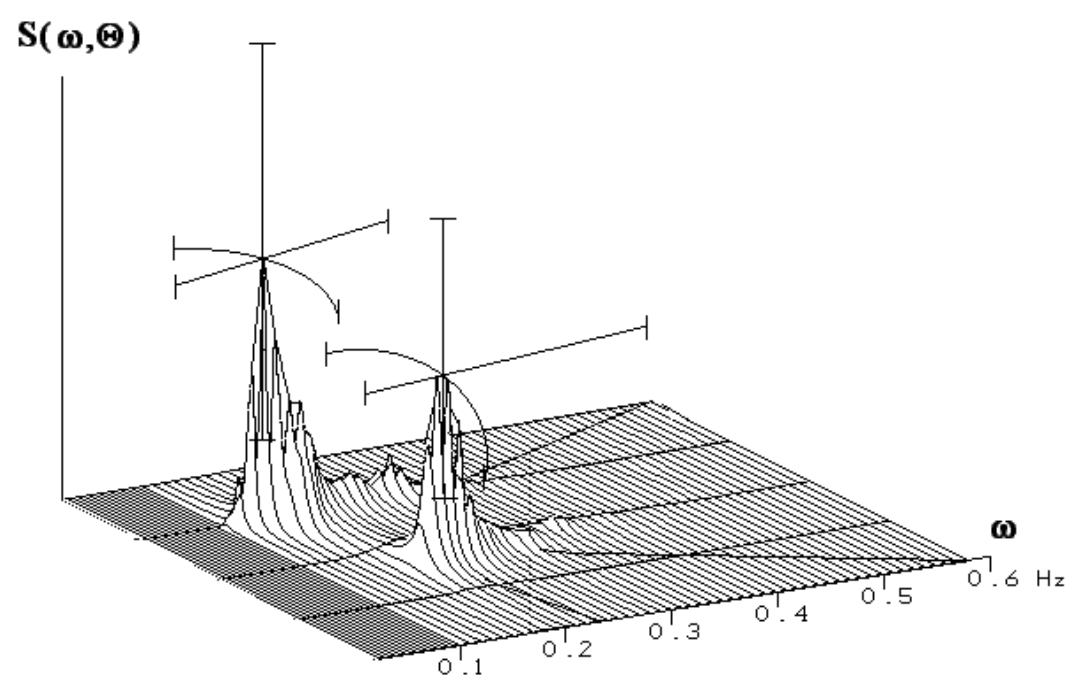

Fig. 1. Frequency-directed climatic spectrum of complex sea. North-Eastern part of the Black Sea

Hence, expressions (2) and (3) do not require comments only for $\mathrm{p}(\bullet)$. Addition operation in (2) is carried out in accordance with "parallelogram rule" for $\vec{V}(\bullet)$. Multiplication operation in (3) is understood as tensor product. Then $m_{\vec{V}}(\bullet)$ is vector, and $K_{\vec{V}}(\bullet)$ is dyadic tensor [15]. Spectrum $\mathrm{S}(\omega, \Theta, \vec{r}, \mathrm{t})$ is function for wave field. Therefore interpretation of $m_{S}(\bullet)$ and $K_{S}(\bullet)$ is obvious only when $(\omega, \Theta)$ are fixed.

$$
S(\omega, \Theta)=S(\omega, \Theta, \Xi)
$$

where $\Xi$ is parameters set $\xi_{j}, j=\overline{1, k}$, forming affine vector. Spectral moments or average values associated with them are used as parameters of observed waves elements. Relations for mean vector, its variance and specific quantiles can be obtained with the help of statistical linearization method. Approach (4) issues from the given function of joint distribution $F_{\Xi}\left(z_{1}, \ldots, z_{k}\right)=P\left\{\xi_{1}<z_{1}, \ldots, \xi_{k}<z_{k}\right\}$ of systems of parameters $\Xi$. In fig. 1 
the example of calculation of an average spectrum $S(\omega, \Theta, \bar{\Xi})$ of complex waves (Northeast part of the Black Sea), and also correspondent probabilistic intervals is shown.

In table 1 characteristics of three functional spaces typical for hydrometeorological fields are shown (scalar, Euclidean vector and affine vector).

Table 1. Functional spaces for hydrometeorological fields description

\begin{tabular}{|c|c|c|c|c|c|}
\hline Object & $\begin{array}{l}\text { Population } \\
\text { mean }\end{array}$ & Variation & Scalar product & $\begin{array}{c}\text { Decompositio } \\
\mathrm{n}\end{array}$ & Examples \\
\hline Scalar $\zeta$ & $E[\zeta]=\int \zeta d \mu$ & $\begin{array}{l}D[\zeta]= \\
E\left[\left(\zeta^{0}\right)^{2}\right]\end{array}$ & $\begin{array}{l}(\zeta, \eta)= \\
\int \zeta \eta d \vec{r} d t\end{array}$ & $\sum_{k} a_{k} \varphi_{k}(\vec{r}, t)$ & $\begin{array}{l}\text { atmosphere } \\
\text { pressure field, } \\
\text { and air } \\
\text { temperature }\end{array}$ \\
\hline $\begin{array}{c}\text { Euclidean } \\
\text { vector }\end{array}$ & $E[\vec{V}]=\left[\begin{array}{l}E[u] \\
E[v]\end{array}\right]$ & $\begin{array}{l}D[\vec{V}]= \\
E\left[\vec{V}^{o} \otimes \vec{V}^{o}\right]\end{array}$ & $\begin{array}{l}\left(\vec{V}_{1}, \vec{V}_{2}\right)= \\
\int u_{1} u_{2} d \vec{r} d t+ \\
\int v_{1} v_{2} d \vec{r} d t\end{array}$ & $\sum_{k} a_{k} \vec{\Psi}_{k}(\vec{r}, t)$ & $\begin{array}{l}\text { wind speed field, } \\
\text { sea currents. }\end{array}$ \\
\hline $\begin{array}{l}\text { Affine } \\
\text { vector }\end{array}$ & $E[\Xi]=\left[\begin{array}{c}E\left[\xi_{l}\right] \\
\cdots \\
E\left[\xi_{n}\right]\end{array}\right]$ & $\begin{array}{l}D[\Xi]= \\
E\left[\Xi \cdot \Xi^{T}\right]\end{array}$ & $\begin{array}{l}(\Xi, H)= \\
\int \operatorname{Tr}\left(\Xi \cdot H^{T}\right) d \vec{r} d t\end{array}$ & $\sum_{k} a_{k}\left[\begin{array}{c}\Phi_{l k}(\vec{r}, t) \\
\cdots \\
\Phi_{n k}(\vec{r}, t)\end{array}\right]$ & $\begin{array}{l}\text { wave parameters } \\
\text { field, } \\
\text { temperature, } \\
\text { salinity and } \\
\text { oxygen in water }\end{array}$ \\
\hline
\end{tabular}

Note: $d \mu=\mu^{\prime}(\zeta) d \zeta$ is a measure defined by density of uninterrupted distribution of scalar $\zeta$.

Operations of addition in (2) and multiplication in (3) define rules of actions with ensemble elements. For the further simplification of model of the analysis let us introduce rules of operations with elements of space $\overrightarrow{\mathbf{r}}$ and time $t$, by means of scalar product. From table 1 it is clear, that only concept of scalar product is obvious to scalar values. For Euclidean and affine vectors it generalizes concept of scalar product both in discrete space and in continuous space.

Spaces with scalar product defined in table 1 will be Hilbert [3]. Hence, in each of them any element can be presented as an infinite converging series on some system of basic elements of this space: scalar functions $\varphi_{k}(\vec{r}, t)$, Euclidean $\vec{\Psi}_{k}(\vec{r}, t)$ or affine $\Phi_{k}(\vec{r}, t)$ vectors - functions. Let us use this decomposition as fundamental way for model of statistical analysis simplification and reduction of dimension. They allow to proceed from scalar or vector function to an accounting set of scalar coefficients, i.e. to replace model of stochastic function by system of random values.

\section{Statistical Representation of Time-Spatial Metocean Fields}

Decomposition on basic elements in finite dimensional space is one of classical MSA procedures [2]. Decomposition coefficients $a_{k}$ are called canonical variable. The principal components explaining general variability, factor loadings determining correlation structure, and canonical correlations representing degree of interrelation between two objects are considered depending on the purposes decomposition. The problem of obtaining canonical variables in the analysis of continuous hydrometeorological fields $\eta(\vec{r}, \mathrm{t})$ was traditionally solved by their representation as system of random values $\mathrm{H}=\left\{\eta_{1}, \ldots, \eta_{\mathrm{n}}\right\}$ in characteristic points $\left\{\vec{r}_{\mathrm{i}}\right\}$ or/and at time 
moments $\left\{\mathrm{t}_{\mathrm{i}}\right\}, i=\overline{1, n}$ with correlation matrix $K_{H}=E\left[H \cdot H^{T}\right]$. Classical procedures of matrix algebra were applied for transition to canonical basis [5].

In fig. 2 as an example spatial and time correlation functions of surface atmospheric pressure field are shown. From the figure it is clear, that they decrease slowly enough; the degree of coherence between rather far points is high. Hence, it is possible to speak about quasi-homogeneous areas. In this case using of $\eta_{i}, \eta_{j}$ values in $H_{n}$ in close points $\left(\vec{r}_{i}, \vec{r}_{j}\right)$ results in multicollinearity and estimation of correlation matrix $\mathrm{K}_{\mathrm{H}}$ appears poorly worded and numerically singular. In generalizing work [1] it is noted that now neither quantitative criteria of multicollinearity, nor universal methods of its elimination by means of matrix algebra exist.
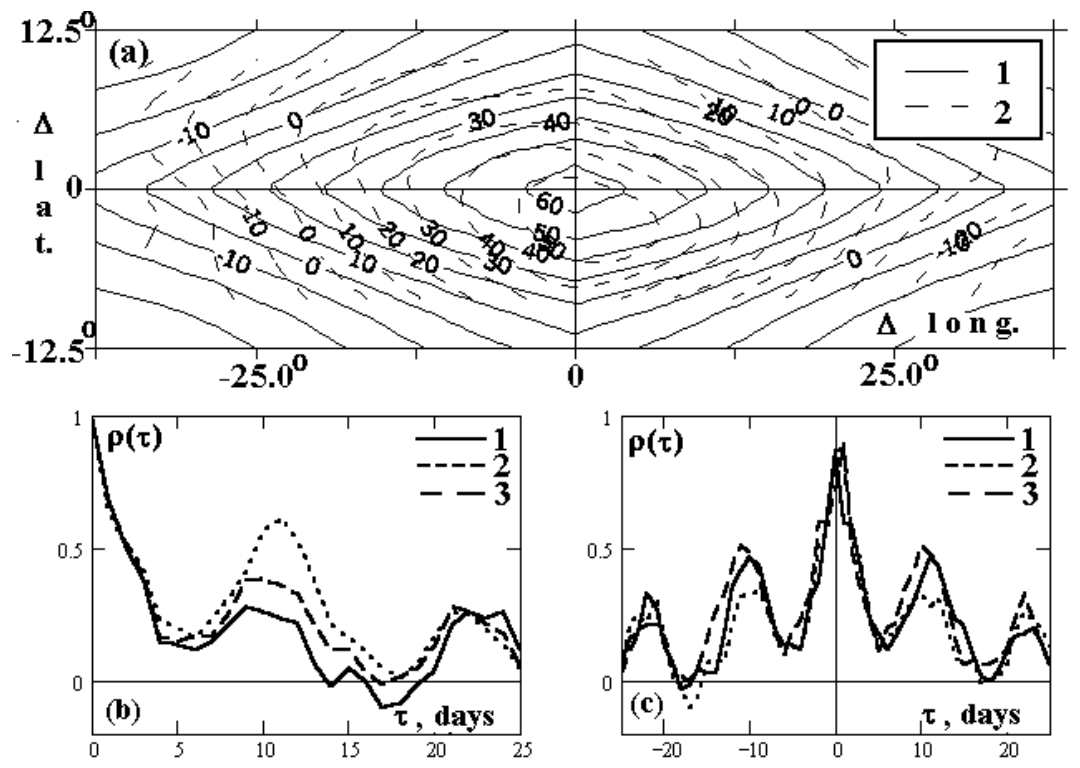

Fig. 2. Estimations of spatial and time correlation functions of pressure field. a) - spatial covariation function $\mathrm{K}_{\mathrm{p}}(\mathrm{x}, \mathrm{y}, \tau)\left(\mathrm{hPa}^{2}\right)$ of pressure field over the Barents Sea in homogeneous approximation: $1-\tau=0,2-\tau=24 \mathrm{~h}$. (b) -autocorrelation functions of pressure in characteristic points over the Barents Sea. $1-\left(75^{\circ} \mathrm{N}, 30^{\circ} \mathrm{E}\right), 2-\left(75^{\circ} \mathrm{N}, 50^{\circ} \mathrm{E}\right), 3-\left(70^{\circ} \mathrm{N}, 40^{\circ} \mathrm{E}\right) .(\mathrm{c})-$ joint correlation functions of pressure in characteristic points over the Barents Sea. $1-\left(75^{\circ} \mathrm{N}, 30^{\circ} \mathrm{E}\right)$ and $\left(75^{\circ} \mathrm{N}, 50^{\circ} \mathrm{E}\right), 2-\left(75^{\circ} \mathrm{N}, 30^{\circ} \mathrm{E}\right)$ and $\left(70^{\circ} \mathrm{N}, 40^{\circ} \mathrm{E}\right), 3-\left(75^{\circ} \mathrm{N}, 50^{\circ} \mathrm{E}\right)$ and $\left(70^{\circ} \mathrm{N}, 40^{\circ} \mathrm{E}\right)$.

Therefore for canonical variable introduction let us resort directly to methods of decomposition in functional spaces shown in table 1. Karhunen [11] and Loeve [14] show that for scalar stochastic functions statistically orthogonal basis is generated by the homogeneous Fredholm integrated equation with the symmetric positive definite kernel

$$
\int_{\langle r\rangle} K\left(\vec{r}, \vec{r}_{1}, t, \tau\right) \varphi\left(\vec{r}_{1}, \tau\right) d \vec{r}_{l} d \tau=\lambda \varphi(\vec{r}, t)
$$

Spectrum of such kernel is discrete. Application of quadrature methods for (4) solution results in matrix representation without avoiding multicollinearity of the 
problem. Therefore let us use projective (variational) methods [6] for obtaining orthogonal basis. It improves conditionality of the problem due to new appropriate orthogonal basis. Occasionally such method allows to obtain analytical solution for some types of modeling representations of autocorrelation function $\mathrm{K}_{\eta}(\bullet)$ of nonhomogeneous field. For example, one of the elementary models describes nonhomogeneous field (in accordance with variation $\sigma^{2}(\mathrm{t})$ ) with correlation function of $K_{\zeta}(t, s)=\sigma(t) \sigma(s) k(t-s), \quad t, s \in[-1,1]$.kind. Let us assume that $\sigma(\bullet)=\sigma_{0}+\beta t$ and $k(t-s)=1-|t-s| / 2$. Then asymptotic expressions for the first two eigenvalues from (5) look as follow:

$$
\begin{aligned}
& \lambda_{I}=\frac{1}{105}\left[27 \beta^{2}+91 \sigma_{0}^{2}+\sqrt{Q}\right], \quad \lambda_{2}=\frac{1}{105}\left[27 \beta^{2}+91 \sigma_{0}^{2}-\sqrt{Q}\right], \\
& Q=169 \beta^{4}+8134 \beta^{2} \sigma_{0}^{2}+2401 \sigma_{0}^{4} .
\end{aligned}
$$

As an example, values $\lambda_{1}, \lambda_{2}$, obtained with the help of quadrature matrix procedure at the given number of knots $\mathrm{N}$ of a uniform grid, and by means of (5) for various combinations $\left(\sigma_{0}, \beta\right)$, are shown in tab. 2. From the tab. we can see that convergence of quadrature (matrix) method is rather slow. By virtue of specificity of substitution of a numerable spectrum by a finite one, such spectrum produces upper estimate for $\lambda_{1}^{*}, \lambda_{2}^{*}$ monotonously converging to true value. Using of analytical solution (5) in all cases gives close enough results, especially for $\lambda_{1}^{*}$. Estimations of $\lambda_{2}^{*}$ are more different from those obtained by matrix method; their specification requires increase order of asymptotic decomposition.

Table 2. Comparison of convergence for matrix (quadrature) method and analytical

\begin{tabular}{|c|c|c|c|c|c|}
\hline \multicolumn{2}{|c|}{ Matrix method } & $\sigma_{0}=1, \beta=0.1$ & $\sigma_{0}=1, \beta=0.3$ & $\sigma_{0}=1, \beta=0.5$ & $\sigma_{0}=1, \beta=0.7$ \\
\hline \multirow{4}{*}{$\mathrm{N}$} & 5 & $\begin{array}{l}1.52 \\
0.59\end{array}$ & $\begin{array}{l}1.66 \\
0.55\end{array}$ & $\begin{array}{l}1.90 \\
0.50\end{array}$ & $\begin{array}{l}2.22 \\
0.48\end{array}$ \\
\hline & 10 & $\begin{array}{l}1.44 \\
0.49\end{array}$ & $\begin{array}{l}1.54 \\
0.46\end{array}$ & $\begin{array}{l}1.72 \\
0.42 \\
\end{array}$ & $\begin{array}{l}1.97 \\
0.40\end{array}$ \\
\hline & 100 & $\begin{array}{l}1.37 \\
0.41\end{array}$ & $\begin{array}{l}1.45 \\
0.39\end{array}$ & $\begin{array}{l}1.59 \\
0.36 \\
\end{array}$ & $\begin{array}{l}1.78 \\
0.34\end{array}$ \\
\hline & 500 & $\begin{array}{l}1.36 \\
0.40 \\
\end{array}$ & $\begin{array}{l}1.44 \\
0.38 \\
\end{array}$ & $\begin{array}{l}1.58 \\
0.36\end{array}$ & $\begin{array}{l}1.77 \\
0.33 \\
\end{array}$ \\
\hline \multicolumn{2}{|c|}{$\begin{array}{l}\text { Analytical } \\
\text { approximation (6) }\end{array}$} & $\begin{array}{l}1.34 \\
0.40 \\
\end{array}$ & $\begin{array}{l}1.42 \\
0.36 \\
\end{array}$ & $\begin{array}{l}1.57 \\
0.30 \\
\end{array}$ & $\begin{array}{l}1.76 \\
0.23 \\
\end{array}$ \\
\hline
\end{tabular}
approximation (6) by variational method.

Each eigenvalue $\lambda_{i}$ sets variance of $i$-th principal component. This is coefficient $a_{i}$, which is determined by inverse transformation of tab. 1

$$
a_{k}=\int_{\langle r\rangle} \zeta(\vec{r}, t) \varphi_{k}(\vec{r}, t) d \vec{r} d t
$$

For vector random field (wind speed or sea currents) $\vec{V}(\vec{r}, t)$ from tab. 1 representation of orthogonal basis at transition to the principal components is ambiguous. Let us consider the problem of obtaining statistically orthogonal basis for 
a random vector field $\vec{V}(\vec{r})=(u, v)$. In this case decomposition coefficients $\mathrm{a}_{\mathrm{k}}$ are not correlated, and the vector basis is the solution of system of homogeneous Fredholm equations

$$
\begin{aligned}
& \int K_{u u}\left(\vec{r}_{1}, \vec{r}_{2}\right) \varphi\left(\vec{r}_{2}\right) d \vec{r}_{2}+\int K_{u v}\left(\vec{r}_{1}, \vec{r}_{2}\right) \psi\left(\vec{r}_{2}\right) d \vec{r}_{2}=\lambda \varphi\left(\vec{r}_{1}\right), \\
& \int K_{v u}\left(\vec{r}_{1}, \vec{r}_{2}\right) \varphi\left(\vec{r}_{2}\right) d \vec{r}_{2}+\int K_{v v}\left(\vec{r}_{1}, \vec{r}_{2}\right) \psi\left(\vec{r}_{2}\right) d \vec{r}_{2}=\lambda \psi\left(\vec{r}_{1}\right),
\end{aligned}
$$

with respect to components $\vec{\Psi}=(\varphi, \psi)$. Orthogonal basis generated by (8) defines operator transformation $\Psi=Q \Phi$ resulting correlative tensor

$$
K_{\vec{V}}\left(\vec{r}_{k}, \vec{r}_{p}\right)=\sum_{k} \sum_{j} \lambda_{i j}\left[\vec{\Psi}_{i}\left(\vec{r}_{k}\right) \otimes \vec{\Psi}_{j}\left(\vec{r}_{p}\right)\right]
$$

in diagonal form:

$$
\Psi K \Psi^{T}=Q \Phi K \Phi^{T} Q^{T}=Q K^{\prime} Q^{T}=\Lambda
$$

where $\Lambda$ is diagonal tensor, composed of variations of decomposition coefficients $\mathrm{a}_{\mathrm{k}}$. Inner orthogonal transformation $\Phi$ from tensor $K$ to $K^{\prime}$ defines turn of principle basis $\left(\vec{e}_{1}^{\prime}, \vec{e}_{2}^{\prime}\right)$ of vector space relative to natural basis $\left(\vec{e}_{1}, \vec{e}_{2}\right)$ in each point $\vec{r}_{k}$. Tensor-function $K^{\prime}$ can be presented as

$$
K_{k p}^{\prime}\left(\vec{r}_{k}, \vec{r}_{p}\right)=\left[\begin{array}{cc}
\lambda_{1}\left(\vec{r}_{k}, \vec{r}_{p}\right) & \vartheta\left(\vec{r}_{k}, \vec{r}_{p}\right) \\
-\vartheta\left(\vec{r}_{k}, \vec{r}_{p}\right) & \lambda_{2}\left(\vec{r}_{k}, \vec{r}_{p}\right)
\end{array}\right]
$$

where $\lambda_{1}, \lambda_{2}$ are principle axes of covariation tensor between points $\left(\vec{r}_{k}, \vec{r}_{p}\right), \vartheta$ is indicator of rotation (when $\mathrm{k}=\mathrm{p} \vartheta$ is equal zero). Transit to principle basis $\left(\vec{e}_{1}^{\prime}, \vec{e}_{2}^{\prime}\right)$ for each pair $\left(\vec{r}_{k}, \vec{r}_{p}\right)$ allows to consider components of vectors $\vec{V}=(u, v)$ independently. Therefore outer orthogonal transformation $Q$ of tensor $K^{\prime}$ to $\Lambda$ defines rotation of sample axis in fundamental space over area $\langle r\rangle$.

For an explanation of correlation structure of scalar and vector random fields, techniques of factor analysis are used. This is representation of random field $\eta(\vec{r}, t)$ as decomposition on the limited number $m$ of coefficients $a_{k}[8]$, specifying correlation function $\mathrm{K}_{\eta}\left(\vec{r}_{1}, \vec{r}_{2}, t, \tau\right)$ as

$$
K_{\eta}\left(\vec{r}_{1}, \vec{r}_{2}, t, \tau\right)=\sum_{i=1}^{m} \lambda_{i} \varphi_{i}\left(\vec{r}_{1}, t\right) \varphi_{i}\left(\vec{r}_{2}, \tau\right)+k_{\varepsilon}(t, \tau)
$$

Here $k_{\varepsilon}(t, \tau)$ is correlation function describing variability of specific and random factors $\varepsilon(t)$. For scalar random fields basis functions (factor loadings) are defined mostly with the help of principal factors method. It consists in application of equation (5) to correlation function corrected on value $\mathrm{k}_{\varepsilon}(\mathrm{t}, \tau)$ [7].

In hydrometeorology for description of spatial and time connectedness two techniques of factor analysis connected with methods of correlation function 
construction $\bullet_{\eta}\left(\vec{r}_{1}, \vec{r}_{2}, t, \tau\right)$ are traditionally distinguished. The first is the S-technique, when sections $K_{\eta}\left(\vec{r}_{1}, \vec{r}_{2}\right)=M\left[\eta^{0}\left(\vec{r}_{1}, t\right) \eta^{0}\left(\vec{r}_{2}, t\right)\right]_{t}$ of spatial correlation function are considered. Numeration of ensemble elements is defined by time $t$. Otherwise, when $K_{\eta}(t, \tau)=M\left[\eta^{0}(\vec{r}, t) \eta^{0}(\vec{r}, \tau)\right]_{\vec{r}}$, we speak about $T$-technique which explains time connectedness.

As an example let us consider double factor model of average monthly variability of surface level atmosphere pressure field in the North hemisphere, which is constructed with the help of $\mathrm{S}-$ and $\mathrm{T}$ - techniques. In fig. 3 structure of factor loadings is presented for these two techniques.

Within the framework of S-technique the first factor (coefficient $a_{1}$, axis $f_{1}$ ) explains $41 \%$, and defines the influence of processes occurring over the Euroasian continent and the Pacific Ocean. The second factor $a_{2}\left(20 \%\right.$ of variability, axis $f_{2}$ ) defines processes over the North American continent and Western part of the Atlantic Ocean. Western part of the Atlantic region influences both factors approximately equally.

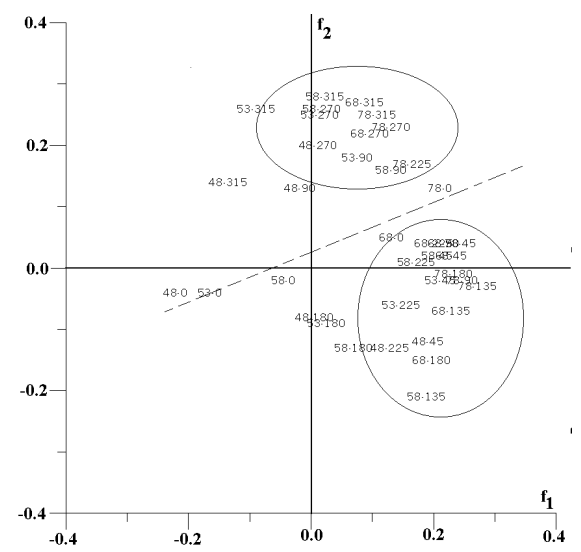

(a)

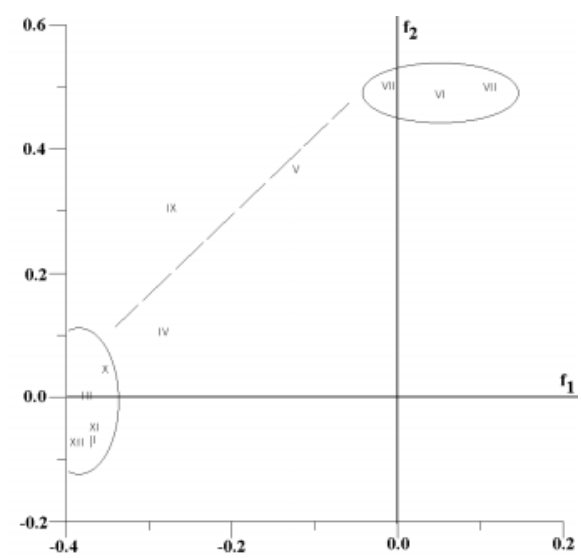

(b)

Fig. 3. Graphic representation of double factor model of correlation structure of atmosphere pressure field over North hemisphere (a) - S-technics: each point is denoted as LATITUDE (N)_LONGITUDE (E) (b) T-technics: each month is denoted by serial number

Within the framework of T-technique in accordance with many years data in the North hemisphere the first factor explains $51 \%$ of variability and characterizes intensity of processes in autumn-winter season (October-February). The second factor (20\% variability) characterizes summer season (June-August). The range corresponding to interseason defines both factors equally.

For description of spatial and time connectedness of system of two dependent random fields $\eta(\vec{r}, \mathrm{t}), \zeta(\vec{r}, \mathrm{t})$ or time connectedness of two spatial areas $\eta\left(\vec{r}_{l}, \mathrm{t}\right)$, $\eta\left(\vec{r}_{2}, \mathrm{t}\right)$ canonical correlation analysis is used [10]. It allows to explain not only structure of auto correlation functions $K_{\zeta}(\bullet), K_{\eta}(\bullet)$, but also joint correlation function $K_{\zeta \eta}(\bullet)$. For this purpose let us introduce in appropriate functional spaces joint canonical basis functions $\mathrm{c}_{\mathrm{k}}(\vec{r}, \mathrm{t})$ and $\mathrm{d}_{\mathrm{k}}(\vec{r}, \mathrm{t})$ and appropriate canonical variables 


$$
U_{k}(t)=\int_{\langle r\rangle} \eta(\vec{r}, t) c_{k}(\vec{r}, t) d \vec{r}, \quad W_{k}(t)=\int_{\langle r\rangle} \zeta(\vec{r}, t) d_{k}(\vec{r}, t) d \vec{r}
$$

for which value of joint correlation function is

$$
\rho\left(U_{k}(t), W_{k}(t+\tau)\right) \rightarrow \max , \quad E\left[U^{2}\right]=E\left[W^{2}\right]=1 .
$$

for any $\tau \geq 0$. Solution of (13-14) as the problem of conditional optimization in functional space results in system of two uniform integral Fredholm equations:

$$
\left\{\begin{array}{c}
-\lambda \int_{\langle r\rangle} K_{\eta \eta}\left(\vec{r}, \vec{r}_{I}\right) c\left(\vec{r}_{I}\right) d \vec{r}_{I}+\int_{\langle r\rangle} K_{\zeta \eta}\left(\vec{r}, \vec{r}_{I}\right) d\left(\vec{r}_{I}\right) d \vec{r}_{I}=0, \\
\int_{\langle r\rangle} K_{\zeta \eta}\left(\vec{r}, \vec{r}_{I}\right) c\left(\vec{r}_{I}\right) d \vec{r}_{I}-\lambda \int_{\langle r\rangle} K_{\zeta \zeta}\left(\vec{r}, \vec{r}_{I}\right) d\left(\vec{r}_{I}\right) d \vec{r}_{I}=0,
\end{array}\right.
$$

relative to functions $\bullet(\vec{r}), \mathrm{d}(\vec{r})$ for stipulated $\tau$ and eigenvalues $\lambda$. By example of atmospheric pressure field in synoptic range of variability in tab. 3 values of first $\lambda_{1}(\tau)$ and second $\lambda_{2}(\tau)$ functions of canonical correlation for two spatial areas (Northwest of Atlantic and Europe) are given. From the table it is clear, that the functions $\lambda_{i}(\tau)$ decrease slowly.

Table 3. Canonical correlation functions for the Northeast Atlantic and Europe. Synoptic variability. Autumn season (October-November).

\begin{tabular}{|c|c|c|c|c|c|}
\hline$\tau$, days & 0 & 5 & 10 & 15 & 20 \\
\hline$\lambda_{1}(\tau)$ & 0.76 & 0.67 & 0.65 & 0.45 & 0.32 \\
\hline$\lambda_{2}(\tau)$ & 0.46 & 0.41 & 0.37 & 0.28 & 0.19 \\
\hline
\end{tabular}

\section{Conclusions}

1. Application of termohydrodynamic modeling methods has allowed to generalize the diverse data of full-scale observations and to create the information base containing characteristics $\Xi=\left\{\xi_{\mathrm{i}}(\vec{r}, t)\right\}$ of the hydrometeorological phenomena on the regular grid $\vec{r}_{k}$ at the given time moments of $t_{s}$.

2. Hydrometeorological fields at fixed $(\vec{r}, t)$ designated as $(\bullet)$ are considered as elements of various functional spaces: scalar (atmospheric pressure $p(\bullet)$ ), Euclidean vector (wind speed $\vec{V}(\bullet)$ ), function (spectral density of complex waves $S(\omega, \Theta, \bullet)$ ). In these spaces operations of addition (averaging), multiplication of elements and scalar product have various interpretation.

3. For the description of variability of spatial-time fields canonical variables as coefficients of decomposition in appropriate Hilbert spaces are used. To avoid of multicollinearity basis functions of decomposition are determined by the solution of appropriate Fredholm equations with the help of variational methods. 


\section{Acknowledgement}

This work is supported by grant INTAS Open 1999 N666.

\section{References}

1. Aivazian S.A., Mkhitarian V.S. Applied statistics and essentials of econometrics. Moskow, Book-Publishing Association UNITY, 1998 (in Russian).

2. Anderson T.W. An introduction to multivariate statistical analysis ( $2^{\text {nd }}$ Ed.) New York, John Wiley, 1984.

3. Balacrishnan, Applied functional analysis. NewYork, John Wiley, 1980

4. Brillinger D.R. Time series. Data analysis and theory. NewYork, Holt, Rinehart and Winston Inc., 1975.

5. Golube G.H., Van Loan C.F. Matrix computations ( $2^{\text {nd }}$ ed.)., London, John Hopkins University Press, 1989.

6. Gould S.H. Variational methods for eigenvalue problems. University of Toronto Press, 1957.

7. Jonson R.A., Wichern D.W. Applied multivariate statistical analysis ( ${ }^{\text {rd }}$ Ed.). Prentice Hall Inc., 1992.

8. Joreskog K.G. Factor analysis by least squares and maximum likelyhood. In: Statistical methods for digital computers. New York, John Wiley, 1975.

9. Hansen I.S. Long-term 3-D modelling of stratification and nutrient cycling in the Baltic sea. Proc. of III BASYS Annual Science Conf., September 20-22, 1999, pp. 31-39.

10. Hotelling H. Relations between two sets of variables. Biometrika, 28, 1936, pp. 321-377.

11. Kahrunen K. Uber lineare Methoden in der Wahrscheinlichkeitsrechnung. Ann. Acad. Sci. Fenn., 37, 1947

12. Kalnay E., M. Kanamitsu, R. Kistler, W. Collins, D. Deaven, L. Gandin, M. Iredell, S. Saha, G. White, J. Woollen, Y. Zhu, A. Leetmaa, R. Reynolds, M. Chelliah, W. Ebisuzaki, W.Higgins, J. Janowiak, K. C. Mo, C. Ropelewski, J. Wang, R. Jenne, D. Joseph. The NCEP/NCAR 40-Year Reanalysis Project. Bulletin of the American Meteorological Society, $\cdot 3$, March, 1996.

13. Komen G.L., Cavaleri L., Donelan M., Hasselmann K., Hasselmann S., Janssen P. Dynamics and modelling of ocean waves. Cambridge University Press., 1994.

14. Loeve M. Fonctions aleatories de second odre. C.R. Acad. Sci. 220, 1945.

15. Lopatoukhin L.J., Rozhkov V.A., Bukhanovsky A.V. The main problems of wind and wave statistics, based on spectral modelling data. Proc. of Coastal Wave Meeting, September, 25-28, Barselona, Spain, 2000, paper 7.5.

16. Rozhkov V.A., Trapeznikov Yu.A. Probabilistic modelling of oceanological processes. S.Petersburg, Hydromet. P.H., 1990 (in Russian).

17. Ocean wave modeling. Plenum Press. NewYork, 1985

18. Tolman H.L. A third-generation model for wind waves on slowly varying, unsteady and inhomogeneous depths and current //J.Phys.Ocean., 1991, vol.21, N6, pp.782-797.

19. Van Vledder G.Ph., de Ronde J.G. Stive M.J.F. Performance of a stectral wind-wave model in shallow water //Proc. $24^{\text {th }}$ Int. Conf. Coast. Eng. ASCE, 1994, pp.753-762

20. Benoit M., Marcos F., Becq F. Development of third-generation shallow water wave model with unstructured spatial meshing //Proc. $25^{\text {th }}$ Int.Conf.Coast.Eng. ASCE, 1996

21. Lavrenov I.V. Mathematical modeling of wind waves in spatial inhomogeneous ocean. St.Petersburg, P.H. Gidrometeoizdat, 1998

22. Ris R.C. Spectral modeling of wind waves in coastal areas //Communication on Hydraulic and Geotechnical Engineering, June - TUDelft, 1997, N97-4 